\title{
Wick rotation and supersymmetry
}

\author{
Arthur J. Mountain * \\ The Blackett Laboratory, Imperial College, Prince Consort Road, \\ London SWY 2BZ, United Kingdom. \\ E-mail: 'A. Mountain@ic.ac.uk'
}

ABSTRACT: Wick rotation of supersymmetric theories is studied. In order to do this a comprehensive prescription for the Wick rotation of spinor fields is presented. This allows the consistent Wick rotation of the supersymmetry algebra and leads eventually to the Wick rotation of supersymmetric field theories. Two complementary descriptions of the Euclidean theory are found. Either it involves "doubled" spinor fields and is manifestly covariant under the Euclidean tangent space group or the spinor fields are subject to a nonlocal projection which recovers the physical degrees of freedom but breaks the tangent space symmetry group. This procedure is then applied to the Wess-Zumino model and eleven-dimensional supergravity.

\section{Introduction}

Wick rotation has its history in the regularisation of quantum field theories. The path integral weights paths with $e^{-i S}$ where $S=\int d^{4} x \mathcal{L}(x)$ is the action. Paths which correspond to large action are suppressed as the exponential becomes highly oscillatory. A Wick rotation is an analytic continuation of the time co-ordinate

$$
t \rightarrow-i t
$$

The aim is to make the action imaginary so that the analytic continuation of $e^{-i S}$ has a real, negative exponent so that the path integral converges.

An area of much recent interest is instanton physics. Instantons are defined in Euclidean space so in order to perform instanton calculations in a "realistic" field theory, one must first Wick rotate the theory. A particular example which has generated much interest recently is instanton cosmology [i]1]. This describes the creation of a universe as an instanton which, at a certain size, continues into a Lorentzian spacetime. It is tempting to try to create this scenario inside M-theory in which case one needs to know the Wick rotation of 11-dimensional supergravity to Euclidean space. There is no standard treatment of Wick rotation in the literature. Indeed,

\footnotetext{
${ }^{*}$ Work done in collaboration with K. S. Stelle
}

one can sometimes see different parts of the same theory Wick rotated in different ways. Our aim here is to present a clear prescription for Wick rotation in the presence of supersymmetry.

The difficulty in performing a Wick rotation on a theory containing spinors is that the representations of spinors change as the signature of the spacetime changes. The Wick rotation of spinors was first considered by Osterwalder and Schrader [2] from the point of view of constructive QFT. Their aim was to directly construct a field theory in Euclidean space which reproduced the Green's functions of a Minkowski space theory, that is to look for a Euclidean spinor $\Psi$ such that

$$
\left\langle\Psi_{\alpha}(x) \Psi_{\beta}^{*}(y)\right\rangle=\left\langle T \psi_{\alpha}(x) \bar{\psi}_{\delta}(y)\right\rangle \gamma_{\delta \beta}^{0} .
$$

This is not possible as the right hand side is not Hermitian. To solve this they introduced two Euclidean spinors $\Psi^{(1)}$ and $\Psi^{(2)}$ which are independent and correspond to the Wick rotation of $\psi$ and $\bar{\psi}$ respectively. This is the phenomenon of "fermion doubling" which we return to below. This work was extended by Nicolai [3] who used their treatment of Dirac spinors to define Euclidean Majorana spinors. As supersymmetric theories typically contain Majorana fermions this allowed Nicolai to define Euclidean super- 
symmetric field theories.

There have been several other approaches to Euclideanisation of theories involving spinors. Most of these have at their heart the notion of analytically continuing the time co-ordinate in a continuous manner. Thus $(\overline{1} \cdot \overline{1}-\overline{1})$ would be replaced by

$$
t \rightarrow e^{-i \theta} t,
$$

where $\theta$ runs from 0 (Minkowski space) to $\pi / 2$ (Euclidean space). These days we would describe this as a T-duality in a timelike direction, effectively embedding the $D$-dimensional Minkowski theory in a space of signature $(1, D)$ and rotating to a theory in the $D$ spacelike dimensions. This

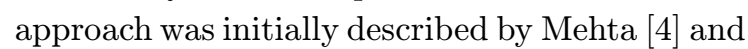
more recently has been studied in [5]. These papers consider the example of $\mathcal{N}=2$ superYang-Mills in four dimensions, rotating this theory to obtain a supersymmetric theory in four Euclidean dimensions which involves Dirac spinors. From the Kaluza-Klein point of view this is all well known; both these theories are dimensional reductions of the $\mathcal{N}=1$ super-Yang-Mills theory in $D=5+1$ dimensions. This procedure is a valid means to arrive at a supersymmetric Euclidean theory (one which was first presented by Zumino [i, $\left.\overline{6}_{1}^{-}\right)$. However we would argue that the aim of Wick rotation is to produce a theory which is a Euclidean-space description of Minkowski-space physics. In this respect, these approaches fail. One clear example of this is that in Zumino's theory there are two scalars which have kinetic terms of opposite sign. This clearly cannot represent Minkowski space physics as the vacuum would be unstable with respect to one of these scalars.

In this paper, we shall investigate the process of Wick rotation in the presence of spinors. In order to do this we start by introducing some of the ideas in the relatively simple setting of a scalar field theory. We then proceed to study spinors in the Wick-rotated theory, following the work of Osterwalder and Schrader and Nicolai. We focus on Majorana spinors as we are interested eventually in the Wick rotation of supersymmetric field theories. We analyse the supersymmetry algebra in Euclidean space and show how this generates supersymmetry in the Wick-rotated theory. Fi- nally we apply these ideas to two theories. The Wess-Zumino model is used as a simple example of a supersymmetric theory. We also discuss Wick rotation of 11-dimensional supergravity. A more detailed exposition of the results presented here will be given in

\section{Wick rotation and the tangent space group}

\section{Scalar field in two dimensions}

In general we will consider spacetimes with coordinates $x=(t, \mathbf{x})$. In this section, we will work in two dimensions so the co-ordinates are $x=$ $\left(t, x_{1}\right)$. The metric is

$$
\eta_{\mu \nu}=\left(\begin{array}{cc}
-1 & 0 \\
0 & 1
\end{array}\right), \quad \eta_{\mu \nu} x^{\mu} x^{\nu}=-t^{2}+x_{1}^{2} .
$$

The basic operation of Wick-rotation is to analytically continue the time co-ordinate as

$$
t \rightarrow-i t \text {. }
$$

Under this, the canonical inner product becomes

$$
\eta_{\mu \nu} x^{\mu} x^{\nu}=-t^{2}+x_{1}^{2} \rightarrow t^{2}+x_{1}^{2}=\tilde{\eta}_{\mu \nu} x^{\mu} x^{\nu},
$$

which involves the expected "Euclidean" metric, $\tilde{\eta}_{\mu \nu}=\operatorname{diag}(1,1)$.

The simplest example of Wick rotation, but one which still has many interesting properties, is the real scalar field $\phi(t, x)$. Under a Wick rotation this becomes

$$
\phi\left(t, x_{1}\right) \rightarrow \Phi\left(t, x_{1}\right)=\phi\left(-i t, x_{1}\right) .
$$

We will define an operation \# which is complex conjugation followed by a Euclidean timereversal. As $\phi$ is real, the Euclidean field $\Phi$ has the following "reality" condition

$$
[\Phi(x)]^{\#} \equiv[\Phi(\theta x)]^{*}=\Phi(x),
$$

where we have introduced the notation $\theta x=$ $\left(-t, x_{1}\right)$. This condition follows directly from the reality condition in Minkowski space. What happens if we apply an $\mathrm{SO}(2)$ transformation? Consider the simple example $\phi(x)=t+x_{1}$. The Euclidean field is $\Phi(x)=-i t+x_{1}$. Under the infinitesimal $\mathrm{SO}(2)$ transformation

$$
\begin{aligned}
\widehat{U}: & \rightarrow U x=x+\delta x, \\
& t \rightarrow t+\epsilon x_{1}, \quad x_{1} \rightarrow x_{1}-\epsilon t,
\end{aligned}
$$


the scalar field transforms into

$$
\begin{aligned}
\Phi^{\prime}(x) & =\widehat{U} \Phi(x)=\Phi(U x) \\
& =-i\left(t+\epsilon x_{1}\right)+\left(x_{1}-\epsilon t\right) .
\end{aligned}
$$

We see that our naive "reality" condition (2.3) is not consistent with the action of $\mathrm{SO}(2)$ in that

$$
\left[\Phi^{\prime}(x)\right]^{\#} \neq \Phi^{\prime}(x) .
$$

At a general point on the $\mathrm{SO}(2)$ orbit the only "reality" condition we can impose is

$$
\left[\widehat{U}^{-1} \Phi^{\prime}(x)\right]^{\#}=\left[\widehat{U}^{-1} \Phi^{\prime}(x)\right] .
$$

In section $\bar{\beta}_{1}$ we will see how to form a covariant version of this condition.

\section{Wick rotation: general prescription}

We shall work in $D$ dimensions. The co-ordinates are $(t, \mathbf{x})$ and we shall analytically continue $t$ as in (2.1). This procedure requires a choice of $t$ and breaks Lorentz covariance. Hence the only part of the $\mathrm{SO}(1, D-1)$ tangent space group which commutes with Wick rotation is the $\mathrm{SO}(D-1)$ group which preserves $t$. The implication of this is that the process of Wick-rotation should be viewed in the following way:

- Start from a theory in D-dimensional Minkowski space with co-ordinates $x^{\mu}$, $\mathrm{SO}(1, D-1)$ symmetry and metric

$$
\eta_{\mu \nu}=\operatorname{diag}(-1,1, \ldots, 1) .
$$

- Choose a preferred time co-ordinate $t$ such that we have co-ordinates $\left(t, x^{k}\right)$. This is a gauge-fixing up to the $\mathrm{SO}(D-1)$ group which acts on the $x^{k}$.

- Perform the Wick-rotation $t \rightarrow-i t$.

- The result is a theory in D-dimensional Euclidean space but a theory which is gaugefixed. By this we mean that the only allowed tangent space rotations are the $\mathrm{SO}(D-1)$ group inherited from the Minkowski theory.

However, this is clearly not the full story. The inner product $\tilde{\eta}_{\mu \nu} x^{\mu} x^{\nu}=\delta_{\mu \nu} x^{\mu} x^{\nu}$ is invariant under $\mathrm{SO}(D)$ whereas the prescription above only gives us an $\mathrm{SO}(D-1)$ symmetry group. In the next section we will show how, by introducing extra degrees of freedom, this can be extended to the full $\mathrm{SO}(D)$ group required for a covariant Euclidean theory. We shall now work in four dimensions, although the generalisation to higher dimension is clear.

\section{Spinor fields}

The added complexity when dealing with spinors is that one has to be careful which representations are allowed. In $3+1$ dimensions, the minimal spinor can be chosen to be Majorana or Weyl while in four Euclidean dimensions we only have a Weyl spinor. See the Appendix for Clifford algebra and spinor conventions.

\section{Dirac spinors}

To define Dirac spinors in the Euclidean theory, we start from the Minkowski spinor $\psi$ and its conjugate $\bar{\psi}=\psi^{\dagger} \gamma^{0}$. Using properties of the $\gamma$-matrices we can see that the $\mathrm{SO}(1, D-1)$ symmetry of the Minkowski theory acts on these as

$$
\delta \psi=\frac{1}{4} \omega_{\mu \nu} \gamma^{\mu} \gamma^{\nu} \psi, \quad \delta \bar{\psi}=-\bar{\psi} \frac{1}{4} \omega_{\mu \nu} \gamma^{\mu} \gamma^{\nu} .
$$

Let us consider the analytic continuation of $\psi$ and $\bar{\psi}$ separately:

$$
\begin{aligned}
& \psi(t, x) \rightarrow \Psi^{(1)}(t, x)=\psi(-i t, x) \\
& \bar{\psi}(t, x) \rightarrow \Psi^{(2)}(t, x)=[\psi(i t, x)]^{\dagger} \gamma^{0} .
\end{aligned}
$$

Before applying a Lorentz transformation we have

$$
\Psi^{(2)}(x)=\left[\Psi^{(1)}(x)\right]^{\#} \gamma^{0}=\left[\Psi^{(1)}(\theta x)\right]^{\dagger} \gamma^{0} .
$$

Following the treatment of the scalar field, we have defined the action of \# on spinors as Hermitian conjugation followed by a Euclidean timereversal. This relationship is not $\mathrm{SO}(4)$-covariant. In order to proceed to an $\mathrm{SO}(4)$ covariant theory, let us define the operator $I$ by

$$
\begin{aligned}
& I \Psi_{\alpha}^{(2)}(x)=\left[\Psi_{\beta}^{(1)}(\theta x)\right]^{\dagger} \gamma_{\beta \alpha}^{0}, \\
& I \Psi_{\alpha}^{(1)}(x)=\left[\Psi_{\beta}^{(2)}(\theta x)\right]^{\dagger} \gamma_{\beta \alpha}^{0} .
\end{aligned}
$$

$I$ is simply an operator which rewrites $\Psi^{(2)}$ in terms of $\Psi^{(1)}$ and vice versa. At a general point 
on the $\mathrm{SO}(4)$ orbit given by $x^{\prime}=U x$, the relationship $\left(\begin{array}{l}1 \\ 3\end{array}-\overline{2}\right)$ must be generalised to

$$
\widehat{U}^{-1} \Psi^{(2)}\left(x^{\prime}\right)=\left[\widehat{U}^{-1} \Psi^{(1)}\left(x^{\prime}\right)\right]^{\#} \gamma^{0} .
$$

Bearing this in mind, we define an involution operator $\Theta$ by

$$
\Theta \equiv \widehat{U} \circ I \circ \widehat{U}^{-1}
$$

In terms of this, we have the discrete symmetry

$$
\Theta \Psi^{(2)}(x)=\left[\Psi^{(1)}(x)\right]^{\#} \gamma^{0},
$$

where $\theta(t, \mathbf{x})=(-t, \mathbf{x})$. The operator $\boldsymbol{\Theta}$ is the Osterwalder-Schrader involution operator. The property $\Theta^{2}=1$ is evident from the definition $(3.4)$. Let us write an $\mathrm{SO}(4)$ element $U$ which rotates $x$ into $x^{\prime}$ by $U\left(x^{\prime}, x\right)$. The action of $\boldsymbol{\Theta}$ is

$$
\begin{aligned}
\Theta U\left(x^{\prime}, x\right) & =U\left(\theta x^{\prime}, \theta x\right), \\
\Theta \Phi(x) & =[\Phi(x)]^{\#}=[\Phi(\theta x)]^{*}, \\
\Theta \Psi_{\alpha}^{(1)}(x) & =\left[\Psi_{\beta}^{(2)}(x)\right]^{\#} \gamma_{\beta \alpha}^{0}, \\
\Theta \Psi_{\alpha}^{(2)}(x) & =\left[\Psi_{\beta}^{(1)}(x)\right]^{\#} \gamma_{\beta \alpha}^{0} .
\end{aligned}
$$

Included above is the covariant treatment of the real scalar field which was mentioned in section We now have two different descriptions of Wick rotated spinor fields. On one hand, we can work with doubled spinors, where $\Psi^{(1)}$ and $\Psi^{(2)}$ are the Wick rotation of $\psi$ and $\bar{\psi}$ and have a theory which is manifestly $\mathrm{SO}(4)$ covariant in the manner described above. On the other hand, we can impose the involution (3.6i) as a nonlocal projection on the fields, for example setting $\Psi_{\alpha}^{(1)}(x)=\left[\Psi_{\beta}^{(2)}(x)\right]^{\#} \gamma_{\beta \alpha}^{0}$. This halves the degrees of freedom (recovering the degrees of freedom of the Minkowski spinors) but breaks $\mathrm{SO}(4)$ covariance.

\section{Hermiticity of the action}

In general, a theory in Minkowski space will have a Hermitian action. We have seen above that when a theory is mapped into Euclidean space using a Wick rotation, Hermiticity is lost. The corresponding symmetry of the Euclidean action is Osterwalder-Schrader positivity, generated by the involution $\boldsymbol{\Theta}$. Using the action of $\boldsymbol{\Theta}$ defined by $\left({ }_{3}^{3} \cdot \overline{6}\right)$, the statement of Osterwalder-Schrader positivity of the Euclidean action is

$$
\boldsymbol{\Theta} \mathcal{L}(x)=[\mathcal{L}(x)]^{\#}=[\mathcal{L}(\theta x)]^{\dagger} .
$$

As an example of this, we know that, in Minkowski space, the combination $\bar{\psi} \phi \psi$ is real and could appear as a Yukawa interaction term in a Lagrangian. In the Euclidean theory, we have the corresponding result

$$
\begin{aligned}
\Theta\left[\Psi^{(2)}(x)\right. & \left.\Phi(x) \Psi^{(1)}(x)\right] \\
= & {\left[\Psi^{(2)}(\theta x) \Phi(\theta x) \Psi^{(1)}(\theta x)\right]^{\dagger} }
\end{aligned}
$$

Of course, all this discussion is using the doubled, $\mathrm{SO}(4)$-covariant spinor fields. If we are prepared to break $\mathrm{SO}(4)$ covariance then, after imposing the nonlocal projection on all fields, we have the reality condition $[\mathcal{L}(x)]^{\#}=\mathcal{L}(x)$.

\section{Majorana spinors}

We wish eventually to perform Wick rotations on supersymmetric field theories. Such theories typically contain Majorana spinors. These are defined by equation (1.A. which can be written (in Minkowski space) as

$$
\psi^{\dagger} \gamma^{0}=\psi^{T} C
$$

Now we need to define our Wick-rotated spinor fields. Using the spinor fields at imaginary time defined above, we see that the Wick rotation of the Majorana condition as originally given by

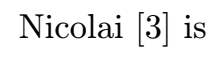

$$
\Psi_{\alpha}^{(2)}(x)=\Psi_{\beta}^{(1)}(x) C_{\beta \alpha} .
$$

The Majorana condition is a reality condition on spinor fields. Following the discussion for Dirac spinors, we see that the action of $\boldsymbol{\Theta}$ is given by

$$
\Theta \Psi_{\alpha}^{(1)}(x)=\gamma_{\alpha \beta}^{0} C_{\beta \gamma}^{\dagger} \Psi_{\gamma}^{(1)}(\theta x)
$$

This is the covariant version of the reality condition one would obtain by simply performing a Wick rotation of $(\overline{3} .9 i)$.

\section{Supersymmetry algebras}

Having defined the image of spinors under Wick rotation, we are now in a position to investigate 
supersymmetry. In four dimensional Minkowski space, the most general supersymmetry algebra is

$$
\left\{Q_{\alpha}, Q_{\beta}\right\}=2 \gamma_{\alpha \beta}^{\mu} \partial_{\mu}+Z_{\mu \nu}\left(\gamma^{\mu} \gamma^{\nu}\right)_{\alpha \beta},
$$

where the central charge $Z_{\mu \nu}$ is real and antisymmetric. There are 10 real degrees of freedom on each side of (4.1).

In Euclidean space, supersymmetry will be generated by a "Euclidean Majorana spinor". Before we impose the Osterwalder-Schrader projection, this is a Dirac spinor $Q^{(1)}$ with $Q^{(2)}=$ $Q^{(1)^{T}} C$. We now have a familiar story. Clearly, only half of the degrees of freedom in $Q^{(1)}$ can be physical but there is no $\mathrm{SO}(4)$-covariant way to identify these degrees of freedom. This means that given a physical state $|\chi\rangle$ there is no $\mathrm{SO}(4)$ covariant way to decompose the 8 supersymmetry generators as

$$
\begin{aligned}
& Q \rightarrow\left\{Q_{M}, \mathcal{Q}_{N}\right\}, M, N=1 \ldots 4 \\
& Q_{M}|\chi\rangle=0, \quad \mathcal{Q}_{M}|\chi\rangle \neq 0 .
\end{aligned}
$$

We are again faced with a choice between $\mathrm{SO}(4)$ covariance and doubled spinors or physical spinors which break this symmetry.

The most general supersymmetry algebra generated by a Dirac spinor in Euclidean space is (omitting the spinor component labels)

$$
\begin{aligned}
\left\{Q, Q^{\dagger}\right\}= & 2 \tilde{\gamma}^{\mu} \partial_{\mu}+i Z^{(0)} \mathbf{1}+Z_{\mu \nu}^{(2)} \tilde{\gamma}^{\mu} \tilde{\gamma}^{\nu} \\
& +i Z_{\mu \nu \rho}^{(3)} \tilde{\gamma}^{\mu} \tilde{\gamma}^{\nu} \tilde{\gamma}^{\rho}+Z^{(4)} \tilde{\gamma}^{5},
\end{aligned}
$$

where all the central charges are real and $Z^{(2)}$ and $Z^{(3)}$ are antisymmetric. There are 16 real degrees of freedom on each side of (4.21). Translating into Osterwalder-Schrader spinors gives the commutator of two Euclidean supersymmetry transformations with parameters $\epsilon_{1}^{(1)}$ and $\epsilon_{2}^{(1)}$ as (c.f. equation 4.1 of [페] $)$

$$
\begin{aligned}
{\left[\delta_{1}, \delta_{2}\right]=} & \epsilon_{2}^{(1) T} C\left(2 \tilde{\gamma}^{\mu} \partial_{\mu}+i Z^{(0)} \mathbf{1}+Z_{\mu \nu}^{(2)} \tilde{\gamma}^{\mu} \tilde{\gamma}^{\nu}\right. \\
& \left.+i Z_{\mu \nu \rho}^{(3)} \tilde{\gamma}^{\mu} \tilde{\gamma}^{\nu} \tilde{\gamma}^{\rho}+Z^{(4)} \tilde{\gamma}^{5}\right) \epsilon_{1}^{(1)}
\end{aligned}
$$

This algebra looks rather different to the supersymmetry algebra in Minkowski space (4.1.1). However, as this is supposed to be a representation of supersymmetry in a Wick-rotated theory, we must impose positivity under the Osterwalder-Schrader involution $\Theta$. This condition sets $Z^{(0)}, Z^{(3)}$ and $Z^{(4)}$ to zero, giving the algebra

$$
\left[\delta_{1}, \delta_{2}\right]=\epsilon_{2}^{(1) T} C\left(2 \tilde{\gamma}^{\mu} \partial_{\mu}+Z_{\mu \nu}^{(2)} \tilde{\gamma}^{\mu} \tilde{\gamma}^{\nu}\right) \epsilon_{1}^{(1)} .
$$

This now looks very similar to $\left(\begin{array}{l}1-1 \\ -1\end{array}\right)$ and indeed has 10 real degrees of freedom on the right hand side. However, the supersymmetry parameter is a Dirac spinor $\epsilon^{(1)}$ which has twice as many degrees of freedom as a (Majorana) supersymmetry parameter in Minkowski space. Only half of these degrees of freedom can be physical but, as mentioned above, there is no $\mathrm{SO}(4)$-covariant way of identifying the physical supersymmetry generators. Following the familiar argument, we would like to impose a non-local projection on the spinors to recover the degrees of freedom of the original Minkowski theory. At first sight, this looks inconsistent; the supersymmetry parameter is constant so the Osterwalder-Schrader projection is simply

$$
\epsilon^{(1)^{T}} C=\epsilon^{(1)^{\dagger}} \gamma^{0},
$$

but this projection is not consistent with the action of $\mathrm{SO}(4)$ given by $(3.1)$. However, we know that after imposing the Osterwalder-Schrader projection we arrive at a gauge-fixed theory (that is a theory which explicitly breaks $\mathrm{SO}(4)$ covariance by involving a reflection in a specific direction labelled by $t$ ). The residual tangent space symmetry is the $\mathrm{SO}(3)$ group which preserves $t$. This is generated by the transformations

$$
\delta \psi=\frac{1}{4} \omega_{j k} \gamma^{j} \gamma^{k} \psi, \quad j, k=1,2,3 .
$$

These transformations are consistent with the projection (4.4). Hence we see that this projection is consistent as long as we remember that we end up in a gauge-fixed theory where the only allowed tangent group transformations are those of the form (4.5i).

After performing an Osterwalder-Schrader

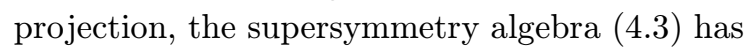
the correct number of degrees of freedom (Majorana spinors on the left, 10 real degrees of freedom on the right).

\section{The Wess-Zumino model}

The Wess-Zumino model is defined in Minkowski 
space by the Lagrangian

$$
\begin{aligned}
\mathcal{L}= & \frac{1}{2}\left[\left(\partial_{\mu} A\right)^{2}+\left(\partial_{\mu} B\right)^{2}+\bar{\psi} \gamma^{\mu} \partial_{\mu} \psi-F^{2}-G^{2}\right] \\
& +g\left[F\left(A^{2}-B^{2}\right)+2 G A B+\bar{\psi}\left(A-\gamma^{5} B \psi\right]\right. \\
& +m\left[F A+G B+\frac{1}{2} \bar{\psi} \psi\right] .
\end{aligned}
$$

The spinor is Majorana, that is $\bar{\psi}=\psi^{T} C=$ $\psi^{\dagger} \gamma^{0}$. This is invariant under the supersymmetry transformations

$$
\begin{array}{lrl}
\delta A=\bar{\epsilon} \psi, & \delta F=-\bar{\epsilon} \gamma^{\mu} \partial_{\mu} \psi, \\
\delta B=\bar{\epsilon} \gamma^{5} \psi, & \delta G=-\bar{\epsilon} \gamma^{5} \gamma^{\mu} \partial_{\mu} \psi, \\
\delta \psi=\partial_{\mu}\left(A-\gamma^{5} B\right) \gamma^{\mu} \epsilon-\left(F+\gamma^{5} G\right) \epsilon
\end{array}
$$

Now let us Wick rotate this theory. We will use the "Euclidean Majorana spinors" $\Psi^{(1)}$ and $\epsilon^{(1)}$, with $\Psi_{\alpha}^{(2)}=C_{\alpha \beta} \Psi_{\beta}^{(1)}$ and $\epsilon_{\alpha}^{(2)}=C_{\alpha \beta} \epsilon_{\beta}^{(1)}$. There is no reality condition on $\Psi^{(1)}$ or $\epsilon^{(1)}$. The Lagrangian becomes

$$
\begin{aligned}
\tilde{\mathcal{L}}= & \frac{1}{2}\left[\left(\partial_{\mu} A\right)^{2}+\left(\partial_{\mu} B\right)^{2}\right. \\
& \left.+\Psi^{(2)} \tilde{\gamma}^{\mu} \partial_{\mu} \Psi^{(1)}-F^{2}-G^{2}\right] \\
& +m\left[F A+G B+\frac{1}{2} \Psi^{(2)} \Psi^{(1)}\right] \\
& +g\left[F\left(A^{2}-B^{2}\right)+2 G A B\right. \\
& \left.+\Psi^{(2)}\left(A-\gamma^{5} B\right) \Psi^{(1)}\right] .
\end{aligned}
$$

where $\tilde{\gamma}^{\mu}=\left(-i \gamma^{0}, \gamma^{k}\right)$ are the Euclidean Dirac matrices. This Lagrangian is invariant under

$$
\begin{aligned}
& \delta A=\epsilon^{(2)} \Psi^{(1)}, \quad \delta F=-\epsilon^{(2)} \tilde{\gamma}^{\mu} \partial_{\mu} \Psi^{(1)}, \\
& \delta B=\epsilon^{(2)} \tilde{\gamma}^{5}, \quad \delta G=-\epsilon^{(2)} \tilde{\gamma}^{5} \tilde{\gamma}^{\mu} \partial_{\mu} \Psi^{(1)} \\
& \delta \Psi^{(1)}=\partial_{\mu}\left(A-\tilde{\gamma}^{5} B\right) \tilde{\gamma}^{\mu} \epsilon^{(1)}-\left(F+\tilde{\gamma}^{5} G\right) \epsilon^{(1)} .
\end{aligned}
$$

Now there are some important points to make

- There are twice as many degrees of freedom in the "Euclidean Majorana spinors" $\Psi^{(1)}$ and $\epsilon^{(1)}$ as in a Majorana spinor in Minkowski space.

- In Euclidean space, the supersymmetry variations of $A, B, F, G$ are not real so it makes more sense to regard these as complex scalar fields. Hence all fields have been "doubled" under the Wick rotation.
- The Lagrangian is supersymmetric under the Euclidean transformations. In fact, demonstrating invariance under these transformations is equivalent algebraically to demonstrating supersymmetry of the Minkowski Lagrangian. This is because the fundamental relation $\bar{\psi} \epsilon=\bar{\epsilon} \psi$ among Majorana spinors holds as

$$
\Psi^{(2)} \epsilon^{(1)}=\epsilon^{(2)} \Psi^{(1)},
$$

among Euclidean Majorana spinors.

- The Lagrangian is manifestly $\mathrm{SO}(4)$-covariant and maps as $\tilde{\mathcal{L}}(x) \rightarrow[\tilde{\mathcal{L}}(\theta x)]^{\dagger}$ under $\Theta$.

Following the familiar argument, we can impose a non-local reality condition (Majorana condition) on the scalars (spinors) to recover the degrees of freedom of the original Minkowski theory.

Finally we should look at representations of the supersymmetry algebra in the Wick-rotated theory. Each Euclidean field has double the degrees of freedom of its Minkowski counterpart. As this is true for the supersymmetry parameter $\epsilon^{(1)}$ we would expect the representations of supersymmetry to be twice as large in the Euclidean case. This happens in a rather unusual way; every state in the supersymmetry algebra is "complexified" so that the whole algebra is constructed in terms of complex degrees of freedom.

\section{Eleven-dimensional supergravity}

We will now briefly discuss the application of this

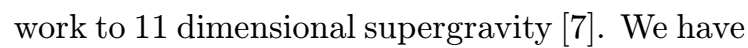
seen above that Majorana spinors can be consistently treated under a Wick rotation. With this in mind, we leave the details to [i] and concentrate for now on the bosonic sector. The bosonic sector of eleven dimensional supergravity is

$$
\begin{gathered}
\mathcal{L}=-\frac{e}{4 \kappa^{2}} R-\frac{e}{48} F_{\mu_{1} \ldots \mu_{4}} F^{\mu_{1} \ldots \mu_{4}} \\
+\frac{2 \kappa}{(12)^{4}} \epsilon^{\mu_{1} \ldots \mu_{11}} F_{\mu_{1} \ldots \mu_{4}} F_{\mu_{5} \ldots \mu_{8}} A_{\mu_{9} \mu_{10} \mu_{11}}
\end{gathered}
$$

In this, $e$ is the determinant of the elfbein, $A$ is a three-form field and

$$
F_{\mu_{1} \ldots \mu_{4}}=4 \partial_{\left[\mu_{1}\right.} A_{\left.\mu_{2} \mu_{3} \mu_{4}\right]} .
$$


This Lagrangian is Hermitian and so is the action $S=\int d^{11} x \mathcal{L}(x)$. This action would appear inside a path integral as $\exp (-i S)$. We now perform a Wick rotation $t \rightarrow-i t$ as described above. In the non-covariant formulation (after the nonlocal projection has been carried out) we have a Euclidean Lagrangian $\tilde{\mathcal{L}}$ which satisfies

$$
[\tilde{\mathcal{L}}(\theta x)]^{\dagger}=\tilde{\mathcal{L}}(x) .
$$

Under the Wick rotation we find

$$
\begin{aligned}
-i S \rightarrow-\tilde{S} & =-i \int_{-\infty}^{\infty}(-i d t) \int d^{10} x \tilde{\mathcal{L}}(x) \\
& =-\int_{-\infty}^{\infty} d t \int d^{10} x \tilde{\mathcal{L}}(x) .
\end{aligned}
$$

Given that $\tilde{\mathcal{L}}$ satisfies $(\overline{6} .2 \bar{j})$ we see that whether a given term of $\tilde{\mathcal{L}}$ gives a real or purely imaginary contribution to $\tilde{S}$ depends on its properties under time-reflection. Let us look closely at two terms in the action. The term $F_{\mu \nu \sigma \tau} F^{\mu \nu \sigma \tau}$ is positive under time-reversal and hence its contribution to $\tilde{S}$ is real. The Chern-Simons term

$$
\epsilon^{\mu_{1} \ldots \mu_{11}} F_{\mu_{1} \ldots \mu_{4}} F_{\mu_{5} \ldots \mu_{8}} A_{\mu_{9} \mu_{10} \mu_{11}}
$$

is negative under a time-reversal and hence its contribution to $\tilde{S}$ is imaginary. This has an important physical meaning; we want gauge transformations to be quantised in both Lorentzian and Euclidean settings. This corresponds to the Chern-Simons term contributing to the path integral as $e^{-i S_{C S}}$ where $S_{C S}$ is real. We have seen that this is indeed the case. Whereas the kinetic term picks up a factor of $-i$ under Wick rotation, the Chern-Simons term in the exponent of $e^{-i S}$ is purely imaginary in both Lorentzian and Euclidean cases.

\section{Conclusion}

We have shown how to treat supersymmetry under a Wick rotation. The key to this is the correct treatment of spinors. There are two possible Euclidean-space descriptions of the Wick rotated theory. One has explicit $\mathrm{SO}(4)$ covariance but involves doubled spinors. A Lagrangian which is Hermitian in Minkowski space has OsterwalderSchrader positivity when Wick rotated. The other is obtained by halving the spinors by a nonlocal projection. This recovers the physical degrees of freedom of the Minkowski theory but breaks the $\mathrm{SO}(4)$ symmetry. We have shown how the supersymmetry algebra is consistent with this prescription for Wick rotation. We have presented a Wick rotated version of 11 dimensional supergravity. It would be interesting to examine the role, discussed in the introduction, that this might play in cosmology.

\section{Acknowledgments}

We thank Tim Evans, Hermann Nicolai and Dan Winder for discussions. AJM thanks the Royal Commission of 1851 for a Research Fellowship. This work was supported in part by PPARC under the SPG grant PPA/G/S/1998/00613.

\section{A. Spinors in four dimensions}

Much of this paper draws on examples in four dimensions. In this Appendix, we list briefly our conventions for spinors in both Minkowski and Euclidean four dimensional space. We use the Clifford algebra conventions of [1] [n] reader to this paper for more information. In all four-dimensional calculations, Greek indices $\mu, \nu, \ldots$ run from 0 to 3 ( 1 to 4 ) when the spacetime is Minkowski (Euclidean). Latin indices $i, j, k, \ldots$ run from 1 to 3 .

\section{Minkowski space}

The Clifford algebra in four-dimensional Minkowski space is

$$
\left\{\gamma^{\mu}, \gamma^{\nu}\right\}=2 \eta^{\mu \nu}=2 \operatorname{diag}(+1,-1,-1,-1)^{\mu \nu}
$$

The Dirac matrices $\gamma^{\mu}$ may be chosen such that

$$
\gamma^{0 \dagger}=\gamma^{0}, \quad \gamma^{k \dagger}=-\gamma^{k}, \quad k=1,2,3 .
$$

In a theory with Majorana spinors, there exist matrices $B$ and $C$ such that

$$
\begin{array}{rll}
\gamma^{\mu *}=B \gamma^{\mu} B^{-1}, & B^{\dagger} B=\mathbf{1}, & B^{T}=B, \\
\gamma^{\mu T}=-C \gamma^{\mu} C^{-1}, & C^{\dagger} C=\mathbf{1}, & C^{T}=-C .
\end{array}
$$

and $B^{T}=C \gamma^{0}$. The Dirac equation is

$$
\left(i \gamma^{\mu} \partial_{\mu}+m\right) \psi(x)=0 .
$$


We can see from the above definitions that if $\psi$ is - a solution of this equation then so is $B_{-}^{-1} \psi_{-}^{*}$. We can then_equate $B_{-}^{-1} \psi_{-}^{*}$ and $\psi_{\text {_via the Majorana }}$ condition

$$
\psi^{*}=B \psi
$$

This can be rewritten as an equality between the -Dirac and_Majorana conjugates

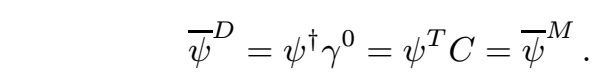

'Imposing the Majorana condition clearly halves the number of degrees of freedom of a spinor.

' Eùcilidēāñ

The Clifford algebra in four-dimensional Euclid- ean space is generated_oby_ $\left\{\tilde{\gamma}_{-}^{\mu}\right\}_{-}$whenere

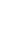

$\tilde{\gamma}^{k}=\gamma^{k}$ for $k=1,2,3, \quad \tilde{\gamma}^{4}=i \gamma^{0}$.

[5] P. van Nieuwenhuizen, A. Waldron, On Euclidean spinors and Wick rotations, Phys. Lett. B 389 (1996) 29, (hep-th/9608174!; A continuous Wick rotation for spinor fields and supersymmetry in Euclidean space, ihep-th/9611043i.

A. Waldron, A Wick rotation for spinor fields: the canonical approach, 'Phys. Lett. B -433! (1998) 369)|hep-th/9702057i.

[6] B. Zumino, Euclidean supersymmetry and the many-instanton problem, 'Phys. Lett. B $\mathbf{6}$ !' (1977) $369^{-1}$

[7] E. Cremmer, B. Julia, J. Scherk, Supergravity theory in 11 dimensions, Phys. Lett. B $\mathbf{\mathbf { 7 }} \overline{\mathbf{6}}_{1}^{\prime}$ $(1978)-409$

[8] T. Kugo, P. K. Townsend, Supersymmetry and the division algebras. 'Nucl. Phys. B $\mathbf{2 2 1}$ (1983) 357!

[9] A. J. Mountain, K. S. Stelle, To appear.

The algebra is

$$
\left\{\tilde{\gamma}^{\mu}, \tilde{\gamma}^{\nu}\right\}=-2 \delta^{\mu \nu}
$$

We have $\tilde{\gamma}^{\mu \dagger}=-\tilde{\gamma}^{\mu}$ for all $\mu$. The matrix $\tilde{\gamma}^{5}=$ $i \tilde{\gamma}^{1} \tilde{\gamma}^{2} \tilde{\gamma}^{3} \tilde{\gamma}^{4}$ is diagonal and antiHermitian.

The charge conjugation matrix is the same as that for Minkowski space and hence satisfies $C^{\dagger} C=\mathbf{1}, C^{T}=-C$ and $\tilde{\gamma}^{\mu T}=-C \tilde{\gamma}^{\mu} C^{-1}$. The Majorana condition is not consistent in Euclidean space as $B^{*} B=-\mathbf{1}$.

\section{References}

[1] S. W. Hawking, N. Turok, Open Inflation Without False Vacua. 'P 1 hys. Lett. B $\mathbf{4 2 5}(1998)$ ', 25ihep-th/9802030"]

[2] K. Osterwalder, R. Schrader, Feynman-Kac formula for Euclidean Fermi and Bose fields, 'Phys. Rev. Lett. 29 (1972)_1423; Euclidean Fermi fields and a Feynman-Kac formula for boson-fermion models, 'Helv. Phys. Acta $\mathbf{4} \mathbf{6}_{1}^{\prime}$ $(1973)-27 \overline{7}$

[3] H. Nicolai, A possible constructive approach to $\left(\text { super }-\phi^{3}\right)_{4},{ }^{\prime}$ Nucl. Phys. B $\mathbf{1 4 0}(1978) 294$.

[4] M. R. Mehta, Euclidean continuation of the Dirac fermion, 'Phys. Rev. Lett. 65 (1990) 1983; Euclideanisation, topological theories, higher dimensions and all that, iPhys. Lett. $\mathbf{B} \mathbf{2 7 4}$ i - _ - 1992) 